\title{
Mother-offspring aggregation in home versus conventional blood pressure in the Tohoku Study of Child Development (TSCD)
}

\author{
Kei ASAYAMA ${ }^{1,2}$, MD, PhD; Jan A. STAESSEN ${ }^{1,3}$, MD, PhD; Katsuhisa HAYASHI ${ }^{4}$, MS; Miki HOSAKA ${ }^{2}$, MS; \\ Nozomi TATSUTA ${ }^{5}$, MS; Naoyuki KUROKAWA ${ }^{5}$, PhD; Michihiro SATOH ${ }^{2}$, MS; Takanao HASHIMOTO ${ }^{6}, \mathrm{PhD}$; \\ Takuo HIROSE ${ }^{2}, \mathrm{PhD}$; Taku OBARA ${ }^{7}, \mathrm{PhD}$; Hirohito $\mathrm{METOKI}^{7}, \mathrm{MD}, \mathrm{PhD}$; Ryusuke INOUE ${ }^{8}, \mathrm{MD}, \mathrm{PhD}$; \\ Masahiro KIKUYA ${ }^{2}, \mathrm{MD}$, PhD; Takayoshi OHKUBO ${ }^{2,9}, \mathrm{MD}, \mathrm{PhD}$; Kunihiko NAKAI ${ }^{5,7}, \mathrm{PhD}$; \\ Yutaka IMAI' ${ }^{2}, \mathrm{MD}, \mathrm{PhD}$; Hiroshi SATOH ${ }^{5}, \mathrm{MD}, \mathrm{PhD}$ \\ ${ }^{1}$ Studies Coordinating Centre, Division of Hypertension and Cardiovascular Rehabilitation, Dept. of Cardiovascular Diseases, University of \\ Leuven, Leuven, Belgium; ${ }^{2}$ Dept. of Planning for Drug Development and Clinical Evaluation, Tohoku University Graduate School of \\ Pharmaceutical Sciences, Sendai, Japan; ${ }^{3}$ Dept. of Epidemiology, Maastricht University, Maastricht, The Netherlands; ${ }^{4}$ Cancer Molecular \\ Biology, Miyagi Cancer Centre, Natori, Japan; ${ }^{5}$ Dept. of Environmental Health Sciences, Tohoku University Graduate School of Medicine, \\ Sendai, Japan; ${ }^{6}$ Dept. of Clinical Pharmacology and Therapeutics, Tohoku University Graduate School of Pharmaceutical Sciences Sendai, \\ Japan; ${ }^{7}$ Dept. of Development and Environmental Medicine, Tohoku University Graduate School of Medicine, Sendai, Japan; ${ }^{8}$ Dept. of Medical \\ Informatics, Tohoku University Graduate School of Medicine, Sendai, Japan; ${ }^{9}$ Dept. of Health Science, Shiga University of Medical Science, \\ Otsu, Japan.
}

Objective Few studies described the home blood pressure (HBP) in young children. Using intrafamilial correlations of blood pressure as research focus, we assessed the feasibility of HBP monitoring in this age group.

Methods We enrolled 382 mothers (mean age 38.8 years) and singletons (7.0 years) in the Tohoku Study of Child Development. We measured their conventional blood pressure (CBP; single reading) at an examination centre. Participants monitored HBP in the morning. We used the OMRON HEM-7080IC for CBP and HBP measurement. In a separate group of 84 children (mean age 7.7 years), we compared blood pressure readings obtained by the OMRON monitor and the Dinamap Pro 100, a device approved by FDA for use in children. We used correlation coefficients as measure of intrafamilial aggregation, while accounting for the mothers' age, body mass index, heart rate and smoking and drinking habits and the children's age, height, and heart rate.

Results Mother-offspring correlations were closer $(P \leq 0.003)$ for HBP than CBP for systolic pressure $[0.28(P<0.0001)$ vs $0.06(P=0.26)]$ and diastolic pressure $[0.28(P<0.0001)$ vs $0.02(P=0.65)]$. The between-device differences (OMRON minus Dinamap) averaged $7.8 \pm 6.0 \mathrm{mmHg}$ systolic and $5.8 \pm 5.5 \mathrm{mmHg}$ diastolic.

Conclusions HBP monitoring is an easily applicable method to assess intrafamilial blood pressure aggregation in young children and outperforms CBP. Validation protocols for HBP devices in young children need revision, because the Korotkoff method is not practicable at this age and there is no agreed alternative reference method.

Keywords Homeblood pressure-intrafamilial aggregation-Korotkoff method-self-measurement-TSCD-young children.

Address for correspondence:

Hiroshi Satoh, MD, PhD, Dept. of Environmental Health Sciences, Tohoku University Graduate School of Medicine, 2-1 Seiryo-cho,

Aoba-ku, Sendai, 980-8575, Japan.

E-mail: h.satoh@nies.go.jp

Received 4 November 2011; accepted for publication 16 January 2012.

\section{INTRODUCTION}

In adults, self-measurement of blood pressure at home is widely used in clinical practice, because compared with conventional blood pressure measurement it has higher reproducibility and provides more accurate risk stratification based on a large consensus about 
diagnostic thresholds ${ }^{1-3}$. Measurement or self-measurement of blood pressure in children by means of semiautomatic or automatic devices is feasible. However, only few studies described blood pressure as measured by automated devices in young children ${ }^{4}$.

The Tohoku Study of Child Development (TSCD) is a cohort study on the potential effects of perinatal exposure to environmental pollutants on the neurobehavioural development of Japanese children ${ }^{5,6}$. This study offered the opportunity to start a new line of longitudinal research into the blood pressure as measured by conventional and automated methods in children growing up from childhood to adolescence. In this first report, we assessed the feasibility of automated oscillometric blood pressure measurement in 7-year-old children. We also compared the intrafamilial aggregation of blood pressure as assessed by an oscillometric technique in an examination centre or by repeated measurements at home.

\section{METHODS}

\section{Study population}

The present study is nested within the longitudinal TSCD. Previous reports describe the study design in detail $^{6}$ as well as eligibility criteria of participants at enrolment ${ }^{5}$. Briefly, mothers in the second trimester of pregnancy and living in Sendai city (from 2001 until $2003, n=599$ ) or in a geographically defined rural area of the Pacific coast (from 2003 until 2005, $n=749$ ) were enrolled in TSCD. Mother-offspring pairs qualified for inclusion in TSCD if the following conditions were met: singleton pregnancy in nulliparous or multiparous women in good health having Japanese as native language and neonates born at term (36-42 weeks of gestation) with birth weight of more than $2400 \mathrm{~g}$ and without congenital anomalies or diseases. The study protocol was approved by the Ethics Committee of the Tohoku University Graduate School of Medicine. Mothers gave written informed consent.

Because the earthquake of 11 March 2011 disrupted data collection in the rural costal area, the present analysis is confined to mothers recruited in Sendai city. Of the 599 mother-neonate pairs originally recruited, 53 were lost to follow-up and 546 participated in a follow-up examination in March 2008 - March 2011, when offspring were approximately 7 years old. Of the 546 mother-offspring pairs followed up, 454 participated in the study of the home blood pressure. We excluded 72 mother-offspring pairs from analysis, because either the conventional or home blood pressure was unavailable in mothers or offspring $(n=20)$, because the home blood pressure had been measured for less than 3 days $(n=33)$, because mothers were taking antihypertensive medication during the survey $(n=3)$, or because not all required characteristics were available for analysis $(n=16)$. Thus, the number of mother-offspring pairs statistically analysed totalled 382.

\section{Blood pressure measurements}

Trained staff measured the conventional blood pressure (one reading) at the examination centre after mothers and offspring had rested for at least 2 minutes in the sitting position. The staff also instructed mothers and offspring how to measure blood pressure at home for two weeks according to the guidelines of the Japanese Society of Hypertension ${ }^{7}$. After having rested in the sitting position for 2 minutes or longer, study participants measured blood pressure at home holding the cuffed arm at the heart level, once in the morning within one hour of waking (after micturition and before breakfast) and once in the evening before going to sleep.

Mothers assisted their children in measuring their home blood pressure. The home blood pressure was the average of the readings obtained over a period of up to 2 weeks.

For blood pressure measurement at the examination centre and at home, we used the OMRON HEM-7080IC (Omron Healthcare Co., Ltd., Kyoto Japan) cuff-oscillometric device ${ }^{8}$. Standard and small cuffs had inflatable bladders of $22 \times 12 \mathrm{~cm}$ and $16 \times 9 \mathrm{~cm}$, respectively, and were used in mothers and offspring depending on upper arm circumference $(22-32 \mathrm{~cm} v s$ under $22 \mathrm{~cm})$. The OMRON HEM-7080IC device is equivalent to the OMRON HEM-705IT. This device passed validation for use in adults ${ }^{9}$ and in children aged from 6 to 16 years ${ }^{10}$. However, to our knowledge, no specific validation studies of the OMRON HEM-7080IC in 7-year-old children are currently available. We therefore compared in a separate group $(n=86)$ of 7 - to 8 -year-old children the OMRON HEM-7080IC with the Dinamap Pro 100 (GE Healthcare, Chalfont St Giles, Buckinghamshire, UK), which is an oscillometric device approved by the Food and Drug Administration (FDA) (http://www.accessdata. fda.gov/cdrh_docs/pdf/k992638.pdf) to monitor the blood pressure in infants and children, and which is commonly used in Japanese hospitals. We applied the protocol of the Japan International Organization for Standardization (ISO) Working Group ${ }^{11}$. The method consists of 4 comparisons between simultaneous readings obtained by the reference and tested device at opposite arms, switching sides each time with the order randomised. 


\section{Anthropometric measurements and other information}

We obtained information on obstetrical data and lifestyle from hospital records and questionnaires administered to mothers. We measured the height and weight of the offspring at follow-up, using a KS-502Gp automatic analyser (Kansai Seiki Co., Ltd., Kusatsu, Japan) with the children wearing light indoor clothes and no shoes. Body mass index was weight in kilogram divided by height in meters squared.

\section{Statistical methods}

For statistical analysis, we used SAS software, version 9.2 (SAS Institute, Cary, NC). We represented the central tendency and spread of continuously distributed variables by the mean and standard deviation. Significance was a 2 -sided $P$-value of 0.05 or less. In the study comparing the OMRON HEM-7080IC and the Dinamap Pro 100 , we applied the Bland and Altman's method.

The current study only included singleton pregnancies and unique mother-offspring pairs. We therefore computed simple product-moment correlation coefficients to express the degree of concordance (positive correlations) or discordance (negative correlations) between mothers and offspring. These analyses were done first without adjustment and next using residuals, in which the contribution of confounders and covariables to the variance of the trait under study was removed. We applied multiple linear regression separately in mothers and offspring to compute the residuals. For the comparison of means and correlation coefficients, we used the Student $t$ test and Fisher $Z$ transformation, respectively.

\section{RESULTS}

\section{Characteristics of study participants}

Gestational weeks at birth (2001-2003) averaged $39.5 \pm 1.2$ with no difference between 182 girls and 200 boys $(P=0.90)$. Length $(48.7 \pm 1.8 v s 49.3 \pm 1.7 \mathrm{~cm})$ and weight (3013 \pm 332 vs $3120 \pm 343$ gram) were significantly lower $(P \leq 0.003)$ in female than male neonates.

The characteristics of mothers and offspring at follow-up (2008-2011) appear in table 1. The mothers' age averaged 38.8 years. Of 382 mothers, 11 (2.9\%) had a history of hypertension, $100(26.2 \%)$ reported regular alcohol intake, and 34 (8.9\%) were smokers. The blood pressure of mothers averaged $109.4 \mathrm{mmHg}$ systolic and $70.2 \mathrm{mmHg}$ on conventional measurement and $109.2 \mathrm{mmHg}$ and $68.0 \mathrm{mmHg}$ on self-measurement in the morning at home [median number of readings 13; interquartile range (IQR), 12-14].

Girls and boys had similar age and anthropometric characteristics $(P \geq 0.43)$. In offspring the median number of readings at home was 13 (IQR, 11-14). The systolic and diastolic blood pressures measured in the examination centre and the diastolic home blood pressure were similar $(P \geq 0.13)$ in girls and boys, averaging $91.6 \pm 9.3 \mathrm{mmHg}, 55.5 \pm 9.2 \mathrm{mmHg}$ and $55.7 \pm 5.8 \mathrm{mmHg}$, respectively. In girls compared with boys $(P \leq 0.002)$, systolic home blood pressure was lower ( $92.6 v s 94.7 \mathrm{~mm}$

Table 1 Characteristics of mothers and offspring

\begin{tabular}{|c|c|c|c|c|}
\hline \multirow[b]{2}{*}{ Variables } & \multirow[t]{2}{*}{ Mothers } & \multicolumn{2}{|c|}{ Offspring } & \multirow[b]{2}{*}{$P$} \\
\hline & & Girls & Boys & \\
\hline Number (n) & 382 & 182 & 200 & \\
\hline Age (years) & $38.8 \pm 4.2$ & $7.0 \pm 0.2$ & $7.0 \pm 0.1$ & 0.79 \\
\hline Height (cm) & $158.2 \pm 4.5$ & $120.0 \pm 4.6$ & $120.2 \pm 4.8$ & 0.62 \\
\hline Weight (kg) & $52.8 \pm 7.3$ & $22.8 \pm 3.1$ & $23.1 \pm 3.0$ & 0.43 \\
\hline Body mass index $\left(\mathrm{kg} / \mathrm{m}^{2}\right)$ & $21.1 \pm 2.7$ & $15.8 \pm 1.5$ & $15.9 \pm 1.3$ & 0.49 \\
\hline \multicolumn{5}{|l|}{ Conventional measurement } \\
\hline Systolic (mmHg) & $109.4 \pm 12.3$ & $91.1 \pm 10.1$ & $91.9 \pm 8.4$ & 0.40 \\
\hline Diastolic (mmHg) & $70.2 \pm 9.0$ & $55.8 \pm 9.3$ & $55.3 \pm 9.2$ & 0.65 \\
\hline Heart rate (bpm) & $67.8 \pm 9.9$ & $82.8 \pm 10.2$ & $78.0 \pm 10.1$ & $<0.0001$ \\
\hline \multicolumn{5}{|l|}{ Home measurement } \\
\hline Systolic (mmHg) & $109.2 \pm 9.9$ & $92.6 \pm 7.1^{*}$ & $94.7 \pm 6.1^{\dagger}$ & 0.002 \\
\hline Diastolic (mmHg) & $68.0 \pm 7.8^{\dagger}$ & $55.2 \pm 5.9$ & $56.1 \pm 5.7$ & 0.13 \\
\hline Heart rate (bpm) & $68.5 \pm 7.6$ & $86.8 \pm 7.0^{\dagger}$ & $82.5 \pm 7.8^{\dagger}$ & $<0.0001$ \\
\hline
\end{tabular}

Values are mean \pm SD. $P$ values are for the comparison between girls and boys. Significance of the difference between conventional and home measurement: ${ }^{*} P<0.05,+P<0.0001$. 
$\mathrm{Hg}$ ), whereas the opposite was the case for heart rate as measured at the examination centre ( $82.8 v s 78.0$ beats per minute) or at home ( $86.8 v s 82.5$ beats per minute).

\section{Mother-offspring correlations for blood pressure}

Table 2 lists the mother-offspring correlations for the conventional and home blood pressures without adjustment and with stepwise adjustment. Adjusted estimates accounted for the mothers' age and body mass index

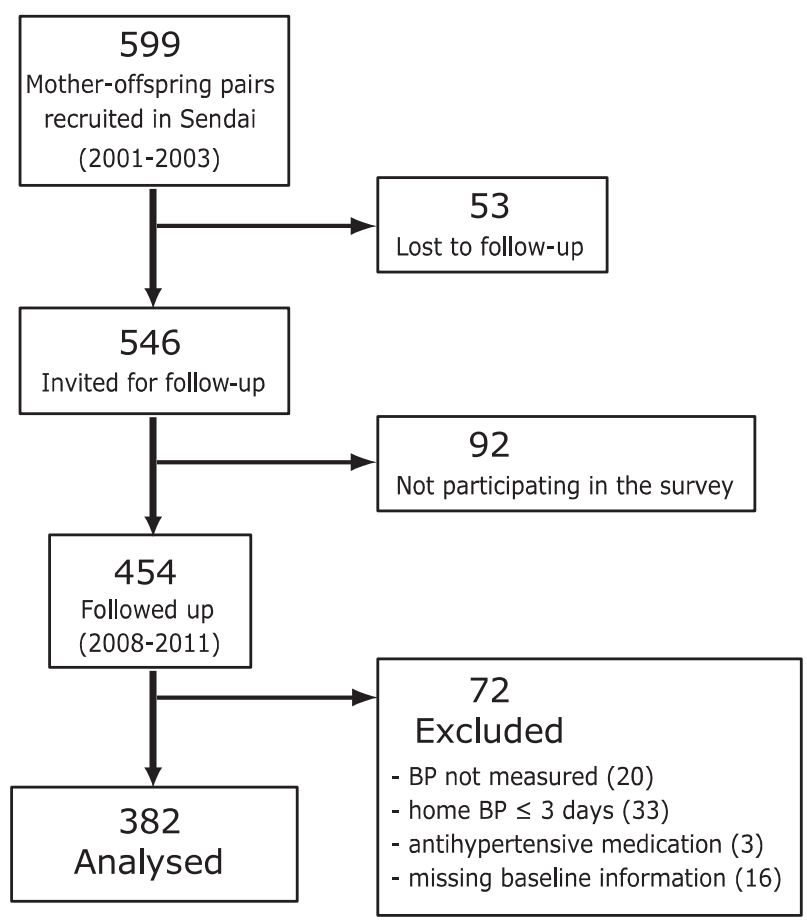

Fig. 1 Flow diagram of study participants. $\mathrm{BP}$ indicates blood pressure. and the children's age and height. Fully adjusted estimates accounted for the same covariables plus smoking and drinking status in mothers and heart rate corresponding with the conventional or home blood pressure (and vice versa) in both mothers and offspring.

In general, unadjusted and adjusted mother-offspring correlations were similar (table 2). With full adjustments applied, the mother-offspring correlations in the conventional systolic and diastolic blood pressures were weak and non-significant $(P \geq 0.39)$, whereas the correlation coefficient for the conventionally measured heart rate was $0.14(P=0.006)$. The fully adjusted mother-offspring correlations for the measurements at home were 0.28 and 0.25 for systolic and diastolic blood pressure $(P<0.0001)$ and 0.17 for heart rate $(P=0.001)$. Mother-offspring correlations in systolic pressure $(P \leq 0.003)$ and diastolic pressure $(P \leq 0.002)$, but not heart rate $(P \geq 0.69)$ were significantly tighter on home than conventional measurement.

Figure 2 shows the mother-offspring correlations for the home measurements stratified by the sex of offspring. For diastolic blood pressure and heart rate, the mother-daughter correlations tended to be higher than the mother-son correlations, but none of the differences between mother-daughter and mother-son correlations reached significance $(P \geq 0.32)$.

\section{Mother-offspring correlations for anthropometric measurements}

The mother-offspring correlations were 0.35 [95\% confidence interval (CI), 0.24-0.42; $P<0.0001]$ for height, 0.29 (CI, 0.19-0.37; $P<0.0001$ ) for weight, and 0.34 (CI, $0.24-0.41 ; P<0.0001$ ) body mass index. Results were similar with adjustments applied for the mothers' age and the children's sex.

Table 2 Intrafamilial mother-offspring correlations

\begin{tabular}{|c|c|c|c|c|c|c|c|c|c|}
\hline \multirow[b]{2}{*}{ Variables } & \multicolumn{3}{|c|}{ Unadjusted } & \multicolumn{3}{|c|}{ Adjusted* } & \multicolumn{3}{|c|}{ Fully adjusted† } \\
\hline & $r$ & $(95 \% \mathrm{Cl})$ & $P$ & $\mathbf{r}$ & $(95 \% \mathrm{Cl})$ & $P$ & $\mathbf{r}$ & $(95 \% \mathrm{CI})$ & $P$ \\
\hline \multicolumn{10}{|c|}{ Conventional measurement } \\
\hline Systolic pressure & 0.06 & $(-0.04,0.16)$ & 0.26 & 0.05 & $(-0.05,0.15)$ & 0.36 & 0.04 & $(-0.06,0.14)$ & 0.39 \\
\hline Diastolic pressure & 0.02 & $(-0.08,0.12)$ & 0.65 & 0.03 & $(-0.07,0.13)$ & 0.57 & 0.03 & $(-0.07,0.13)$ & 0.58 \\
\hline Heart rate & 0.17 & $(0.07,0.27)$ & 0.0007 & 0.13 & $(0.03,0.23)$ & 0.01 & 0.14 & $(0.04,0.24)$ & 0.006 \\
\hline \multicolumn{10}{|l|}{ Home measurement } \\
\hline Systolic pressure & 0.28 & $(0.17,0.36)$ & $<0.0001$ & 0.29 & $(0.19,0.37)$ & $<0.0001$ & 0.28 & $(0.17,0.36)$ & $<0.0001$ \\
\hline Diastolic pressure & 0.28 & $(0.18,0.36)$ & $<0.0001$ & 0.28 & $(0.18,0.37)$ & $<0.0001$ & 0.25 & $(0.15,0.34)$ & $<0.0001$ \\
\hline Heart rate & 0.20 & $(0.10,0.29)$ & $<0.0001$ & 0.16 & $(0.06,0.25)$ & 0.002 & 0.17 & $(0.07,0.26)$ & 0.001 \\
\hline
\end{tabular}

Values are correlation coefficients ( $95 \%$ confidence interval). *Adjusted estimates accounted for the mothers' age and body mass index and the children's age and height. tFully adjusted estimates accounted for the same covariables plus smoking and drinking status in mothers and heart rate corresponding with the conventional or home blood pressure (and vice versa) in both mothers and offspring. 


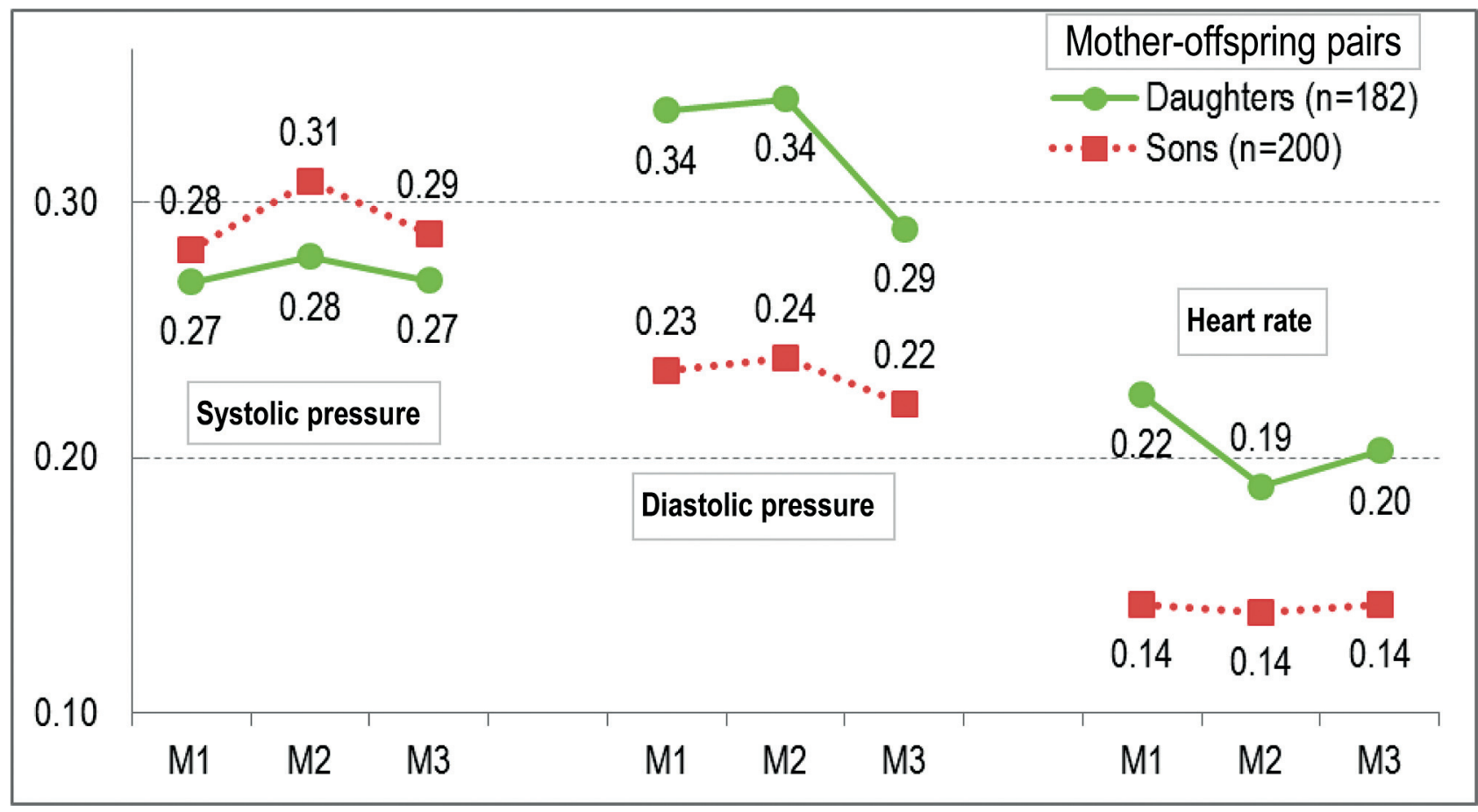

Fig. 2 Plotted values are correlation coefficients in 182 mother-daughter and 200 mother-son pairs.

M1, M2, and M3 refer to unadjusted, adjusted and fully adjusted estimates, respectively. Adjusted estimates accounted for the mothers'age and body mass index and the children's age and height. Fully adjusted estimates accounted for the same covariables plus smoking and drinking status in mothers and heart rate corresponding with the conventional or home blood pressure (and vice versa) in both mothers and offspring. There were no significant differences between mother-daughter and mother-son correlations $(P \geq 0.32)$.

\section{Comparison of devices}

Mean age of the 40 girls and 46 boys participating in the comparison between the Dinamap and the OMROM was $7.67 \pm 0.29(\mathrm{SD})$ years. The difference in the blood pressure readings (OMRON minus Dinamap) averaged $7.8 \pm 6.0 \mathrm{mmHg}$ systolic and $5.8 \pm 5.5 \mathrm{mmHg}$ diastolic. The Bland and Altman plots appear in figure 3.

\section{DISCUSSION}

The key finding of the current study was that the mother-offspring correlations in systolic and diastolic blood pressures, but not heart rate, were significantly higher on home than conventional measurement. The mother-offspring correlations in the conventional blood pressure did not reach statistical significance. In addition, the mother-daughter correlations in the diastolic home blood pressure tended to be slightly higher than the mother-son correlations.

Several previous reports described the familial aggregation of blood pressure ${ }^{12-15}$. However, to the best of our knowledge, no previous studies used automated blood pressure measurement. In a Belgian study published in $1985^{14}$, the parent-offspring correlations were significant for systolic blood pressure in father-son $(r=0.33)$ and mother-daughter $(r=0.24)$ pairs, whereas this was not the case for the other parent-offspring correlations for systolic and diastolic pressure. In this Belgian study ${ }^{14}$, each participant was characterised by the mean of 10 blood pressure readings, 5 readings at each of 2 home visits 2 to 3 weeks apart. As described in detail elsewhere ${ }^{14}$, the observers recording the blood pressure at home were especially trained. Training required at least 4 training sessions and an additional test session. Blood pressure of all members of the same household was measured by the same team of observers, which necessitated adjustment of the intrafamilial correlations for observer bias. The similarity in the mother-offspring correlations in systolic blood pressure in our current study and the previous Belgian study $(0.28 v s 0.24)$ shows that to assess the intrafamilial aggregation of blood pressure automated measurement at home is an easily applicable method, which is much less costly and labour intense than conventional measurement.

The Tecumseh Blood Pressure study compared estimates of blood pressure obtained in the clinic-office setting (average of 2 readings) with self-reported home blood pressure readings (average of 14 readings) taken over 1 week $^{15}$. A trained technician instructed the 

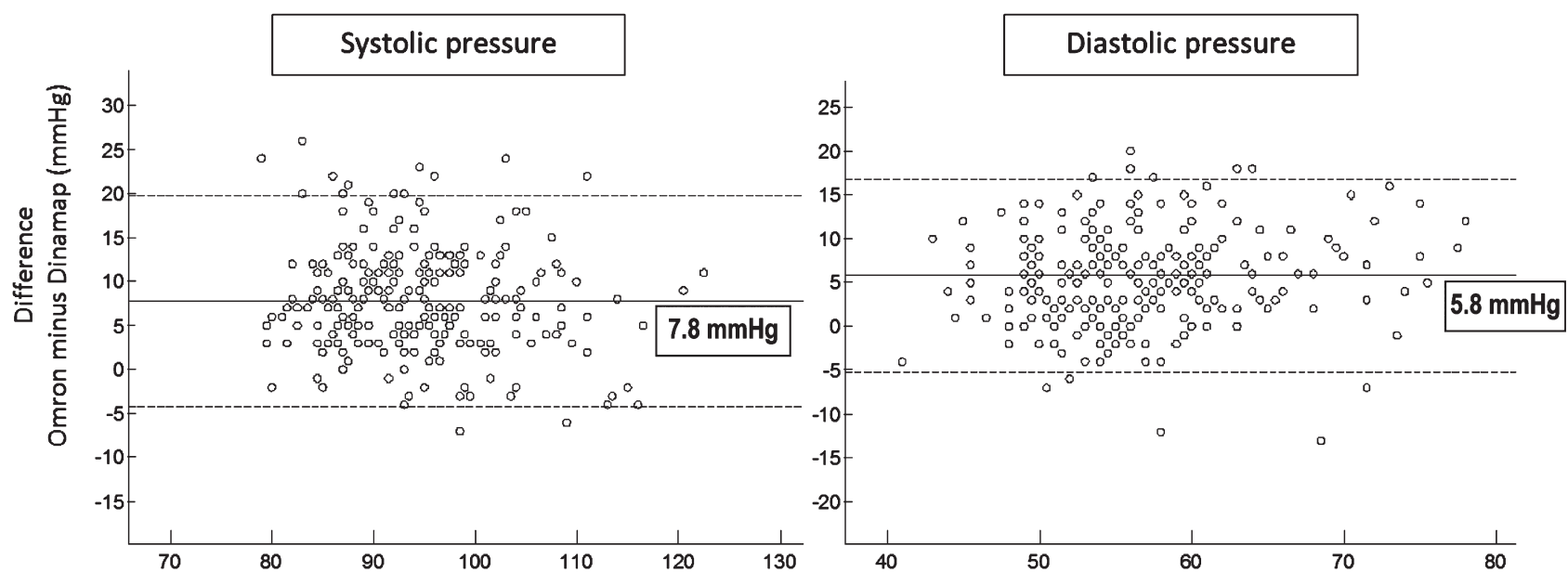

Mean Omron and Dinamap ( $\mathrm{mmHg}$ )

Fig. 3 Bland and Altman plots for the comparison between the OMRON HEM-7080/C and Dinamap Pro 100 devices in 86 7-8 year-old children. Mean differences between paired measurements $\pm 1.96 \mathrm{SD}$ are represented by full and dotted lines, respectively.

participants in self-measurement of the home blood pressure by the Korotkoff technique. Among 380 siblings (average age, 31.4 years), correlation coefficients for the home readings were of the same magnitude as for office readings ( $r=0.23 v s 0.24)$. Thus, in contrast to our current findings, home monitoring of blood pressure by a manual method based on auscultation did not improve estimates of intrafamilial aggregation. Taken together, these observations support the use of automated techniques to assess heritability or intrafamilial aggregation of blood pressure.

Our current results demonstrated that intrafamilial correlation of diastolic home blood pressure as well as home heart rate between mothers and daughters tended to be tighter than that between mothers and sons. In 502 Tokelau Island children ${ }^{12}$, aged 5-14 years, the mother's systolic pressure was the only predictor of the similarity of systolic blood pressure among 210 sibling pairs. Morrison et al. reported that significant mother-daughter correlations in obesity existed regardless of subjects' race $^{16}$. Similarly, in the Belgian study ${ }^{14}$, the correlation coefficients for body weight and body mass index tended to be higher in mother-offspring than in father-offspring pairs, suggesting a closer maternal influence in setting the balance between calorie intake and expenditure in offspring. To what extent factors related to energy metabolism or body weight might influence the motheroffspring aggregation in blood pressure, remains to be elucidated.

The reproducibility of home blood pressure in children, like that in adults, is better than that of conventional blood pressure ${ }^{17}$. Our current results are in keeping with the Arsakeion study, in which the home blood pressure of healthy young children aged 6-12 years was higher than the conventional blood pressure ${ }^{4}$. In the Arsakeion study, the difference between home and conventional blood pressure disappeared in older children and adolescents ${ }^{4}$. Physical activity in children is probably one of the major determinants of blood pressure. In addition, young children might be less likely to follow the instructions for home blood pressure monitoring, in particular observing a resting period before the actual measurement ${ }^{4}$. The observation that girls had lower blood pressure and faster heart rate than boys is in line with the observations in adults ${ }^{18,19}$ and represents a validation of our measurements in children. The mother-offspring correlations in the anthropometric measurements are also in line with those reported in the literature ${ }^{16,20}$.

The present study identified an important issue in the validation of automated devices for home blood pressure measurement in young children. The OMRON 705IT passed validation for use in children ${ }^{10}$ according to the $\mathrm{AAMI}^{21}$ and ESH-IP ${ }^{22}$ criteria. Validation involved simultaneous blood pressure measurements taken by 2 observers (Y-tube connected mercury sphygmomanometers) 4 times in sequence with 3 measurements by the OMRON device. Systolic blood pressure was determined at the first appearance of Korotkoff sounds (phase I) and diastolic blood pressure at the disappearance of sounds (phase V). The study included 197 children. Mean age was $10.6 \pm 2.4$ (SD) years, but ranged from 6 to 16 years. Validation of devices across such a wide age range should probably not be extrapolated to children aged 7 years as enrolled in our current study. Furthermore, the mean height of our study participants was $120.1 \pm 4.7$ (SD) cm. Height in the published validation study ${ }^{10}$ ranged from 
$123 \mathrm{~cm}$ to $186 \mathrm{~cm}$. A crucial issue is that validation requires phase $\mathrm{V}$ diastolic blood pressure to be recorded, which is often impossible in young children, because Korotkoff sounds are often audible until zero cuff pressure $^{23}$. In another validation study, children whose Korotkoff sounds were still audible at a cuff pressure below $10 \mathrm{~mm} \mathrm{Hg}$ were excluded from analysis ${ }^{24}$. Validating diastolic blood pressure against Korotkoff phase IV sounds is not an alternative, because it leads to inconsistencies between and within observers. Lip et al. reported that there was considerable variation on the choice of auscultatory end points for measuring diastolic blood pressure in clinical practice ${ }^{25}$. The current protocols for validation make little provision to address or circumvent these problems.

For the reasons outlined above, we compared, in a separate study, the blood pressure values between OMRON HEM-7080IC and the Dinamap Pro 100, which is widely used in infants ${ }^{26}$ and prepubertal children ${ }^{27}$. The Dinamap is a stationary device approved by FDA. The computational algorithm embedded in the Dinamap was tested against the intra-arterial blood pressure ${ }^{28}$. The bias between the 2 devices averaged $7.8 \mathrm{~mm} \mathrm{Hg}$ systolic and $5.8 \mathrm{~mm} \mathrm{Hg}$ diastolic. However, the difference between the 2 devices was not dependent on level, and it had probably little influence on the estimates of the mother-offspring correlations.

The present study has to be interpreted within the context of its limitations. First, we included only 7-yearold children in our study. Second, we enrolled only one child per family. We did not assess sib-sib or fatheroffspring correlations. Third, we obtained only a single reading of the conventional blood pressure in each participant. Notwithstanding these limitations, our study proved that home monitoring of blood pressure in young children is feasible. Approximately $20 \%$ of children diagnosed as hypertensive by conventional blood pressure measurement at the age of junior high school remain hypertensive 20 years later ${ }^{29}$. We propose that home blood pressure monitoring should become part of the diagnostic work-up and management of children suspected to be hypertensive.

In conclusion, home monitoring of blood pressure is an easily applicable method to assess heritability and intrafamilial aggregation of blood pressure and outperforms conventional blood pressure in this regard. The intrafamilial correlation of blood pressure might be higher in mother-daughter than mother-son pairs, although sufficiently powered studies are necessary to consolidate this observation. Current protocols for the validation of devices for home monitoring of blood pressure in young children need revision.

\section{ACKNOWLEDGEMENTS}

The authors gratefully acknowledge all staff members of the Tohoku Study of Child Development for their valuable contribution to the data collection and database management. Atsushi Sato and Akira Mimura instructed participants how to measure blood pressure. Osamu Shirasaki and Keita Komine, Omron Healthcare, Kyoto, Japan, provided advice for the study comparing devices.

\section{CONFLICT OF INTEREST AND SOURCE OF FUNDING}

This research was funded by a grant from the Ministry of Health, Labour and Welfare, Japan, and the Ministry of the Environment. KA received research support from the Japan Research Foundation for Clinical Pharmacology. The funding sources had no role in the study protocol, in the collection, analysis, and interpretation of data, in the writing of the report, or in the decision to submit the paper for publication. For this reason, the findings and conclusions of this article are solely the responsibility of the authors and do not represent the official views of the above government agencies.

\section{REFERENCES}

1. Ohkubo T, Imai Y, Tsuji I, Nagai K, Kato J, Kikuchi N, Nishiyama A, Aihara A, Sekino M, Kikuya M, Ito S, Satoh H, Hisamichi S. Home blood pressure measurement has a stronger predictive power for mortality than does screening blood pressure measurement: A population-based observation in Ohasama, Japan. J Hypertens 1998; 16: 971-5.

2. Asayama K, Ohkubo T, Kikuya M, Metoki H, Hoshi H, Hashimoto J, Totsune K, Satoh H, Imai Y. Prediction of stroke by self-measurement of blood pressure at home versus casual screening blood pressure measurement in relation to the Joint National Committee 7 classification: the Ohasama study. Stroke 2004; 35: 2356-61.

3. Asayama K, Ohkubo T, Kikuya M, Metoki $\mathrm{H}_{\text {, }}$ Obara T, Hoshi H, Hashimoto J, Totsune K, Satoh H, Imai Y. Use of 2003 European Society of Hypertension-European Society of Cardiology guidelines for predicting stroke using self-measured blood pressure at home: the Ohasama study. Eur Heart J 2005; 26: 2026-31.
4. Stergiou GS, Rarra VC, Yiannes NG. Changing relationship between home and office blood pressure with increasing age in children: the Arsakeion School study. Am J Hypertens 2008; 21: 41-6.

5. Suzuki K, Nakai K, Sugawara T, Nakamura T, Ohba T, Shimada M, Hosokawa T, Okamura K, Sakai T, Kurokawa N, Murata K, Satoh C, Satoh H. Neurobehavioral effects of prenatal exposure to methylmercury and PCBs, and seafood intake: neonatal behavioral assessment scale results of Tohoku study of 
child development. Environ Res 2010; 110: 699-704.

6. Nakai K, Suzuki K, Oka T, Murata K, Sakamoto M, Okamura K, Hosokawa T, Sakai T, Nakamura T, Saito Y, Kurokawa N, Kameo S, Satoh H. The Tohoku Study of Child Development: A cohort study of effects of perinatal exposures to methylmercury and environmentally persistent organic pollutants on neurobehavioral development in Japanese children. Tohoku J Exp Med 2004; 202: 227-37.

7. Ogihara T, Kikuchi K, Matsuoka H, Fujita T, Higaki J, Horiuchi M, Imai Y, Imaizumi T, Ito S, Iwao H, Kario K, Kawano Y, Kim-Mitsuyama S, Kimura G, Matsubara H, Matsuura H, Naruse M, Saito I, Shimada K, Shimamoto K, Suzuki H, Takishita S, Tanahashi N, Tsuchihashi T, Uchiyama M, Ueda S, Ueshima H, Umemura S, Ishimitsu T, Rakugi H. The Japanese Society of Hypertension Guidelines for the Management of Hypertension (JSH 2009). Hypertens Res 2009; 32: 3-107.

8. Coleman A, Steel S, Freeman P, de Greeff A Shennan A. Validation of the Omron M7 (HEM-780-E) oscillometric blood pressure monitoring device according to the British Hypertension Society protocol. Blood Press Monit 2008; 13: 49-54.

9. El Assaad MA, Topouchian JA, Asmar RG. Evaluation of two devices for self-measurement of blood pressure according to the international protocol: the Omron m5-I and the Omron 705IT. Blood Press Monit 2003; 8: 127-33.

10. Stergiou GS, Yiannes NG, Rarra VC. Validation of the Omron 705 IT oscillometric device for home blood pressure measurement in children and adolescents: the Arsakion School Study. Blood Press Monit 2006; 11: 229-34.

11. Shirasaki $O$, Asou $Y$, Takahashi Y. New clinical validation method for automated sphygmomanometer: a proposal by Japan ISO-WG for sphygmomanometer standard. Blood Press Monit 2007; 12: 381-3.

12. Beaglehole R, Salmond CE, Prior IA. A family study of blood pressure in Polynesians. Int J Epidemiol 1975; 4: 217-20.
13. Biron P, Mongeau JG, Bertrand D. Familial aggregation of blood pressure in 558 adopted children. Can Med Assoc J 1976; 115: 773-4.

14. Staessen J, Bulpitt CJ, Fagard R, Joossens JV, Lijnen P, Amery A. Familial aggregation of blood pressure, anthropometric characteristics and urinary excretion of sodium and potassium--a population study in two Belgian towns. J Chronic Dis 1985; 38: 397-407.

15. Jamerson KA, Schork N, Julius S. Effect of home blood pressure and gender on estimates of the familial aggregation of blood pressure. The Tecumseh Blood Pressure Study. Hypertension 1992; 20: 314-8.

16. Morrison JA, Payne G, Barton BA, Khoury PR, Crawford P. Mother-daughter correlations of obesity and cardiovascular disease risk factors in black and white households: the NHLBI Growth and Health Study. Am J Public Health 1994; 84: 1761-7.

17. Stergiou GS, Nasothimiou EG, Giovas PP, Rarra VC. Long-term reproducibility of home vs. office blood pressure in children and adolescents: the Arsakeion school study. Hypertens Res 2009; 32: 311-5.

18. Ueshima $\mathrm{H}$. Explanation for the Japanese paradox: prevention of increase in coronary heart disease and reduction in stroke. J Atheroscler Thromb 2007; 14: 278-86.

19. Morcet JF, Safar M, Thomas F, Guize L, Benetos A. Associations between heart rate and other risk factors in a large French population. J Hypertens 1999; 17: 1671-6.

20. Fuentes RM, Notkola IL, Shemeikka S, Tuomilehto J, Nissinen A. Familial aggregation of body mass index: a population-based family study in eastern Finland. Horm Metab Res 2002; 34: 406-10.

21. White WB. National standard for measurement of resting and ambulatory blood pressures with automated sphygmomanometers. Hypertension 1993; 21: 504-9.

22. O'Brien E, Pickering $T$, Asmar R, Myers $M$, Parati G, Staessen J, Mengden T, Imai Y, Waeber B, Palatini P, Gerin W; Working Group on Blood Pressure Monitoring of the European Society of Hypertension. Working Group on Blood Pressure Monitoring of the European Society of Hypertension International Protocol for validation of blood pressure measuring devices in adults. Blood Press Monit 2002; 7: 3-17.

23. Hammond IW, Urbina EM, Wattigney WA, Bao W, Steinmann WC, Berenson GS Comparison of fourth and fifth Korotkoff diastolic blood pressures in 5 to 30 year old individuals. The Bogalusa Heart Study. Am J Hypertens 1995; 8: 1083-9.

24. Wong SN, Tz Sung RY, Leung LC. Validation of three oscillometric blood pressure devices against auscultatory mercury sphygmomanometer in children. Blood Press Monit 2006; 11: 281-91.

25. Lip GY, Beevers M, Beevers DG, Dillon MJ. The measurement of blood pressure and the detection of hypertension in children and adolescents. J Hum Hypertens 2001; 15: 419-23.

26. Lurbe E, Garcia-Vicent C, Torro I, Fayos JL, Aguilar F, de Llano JM, Fuertes G, Redon J. First-year blood pressure increase steepest in low birthweight newborns. J Hypertens 2007; 25: 81-6.

27. Jin RZ, Donaghue KC, Fairchild J, Chan A, Silink M. Comparison of Dinamap 8100 with sphygmomanometer blood pressure measurement in a prepubertal diabetes cohort. J Paediatr Child Health 2001; 37: 545-9.

28. Ni H, Wu C, Prineas R, Shea S, Liu K, Kronmal R, Bild D. Comparison of Dinamap PRO-100 and mercury sphygmomanometer blood pressure measurements in a population-based study. Am J Hypertens 2006; 19: 353-60.

29. Uchiyama M. Risk factors for the development of essential hypertension: Long-term follow-up study in junior high school students in Niigata, Japan. J Hum Hypertens 1994; 8: 323-5. 